\title{
Lepton angular distributions of Drell-Yan process in pQCD and a geometric approach
}

\author{
Wen-Chen Chang* \\ Institute of Physics, Academia Sinica, Taipei 11529, Taiwan
}

\section{Randall Evan McClellan}

Thomas Jefferson National Accelerator Facility, Newport News, VA 23606, USA

\section{Jen-Chieh Peng}

Department of Physics, University of Illinois at Urbana-Champaign, Urbana, Illinois 61801, $U S A$

\section{Oleg Teryaev}

Bogoliubov Laboratory of Theoretical Physics, JINR, 141980 Dubna, Russia

The lepton angular distributions of the Drell-Yan process in the fixed-target experiments are investigated by NLO and NNLO perturbative QCD. We present the calculated angular parameters $\lambda, \mu, v$ and the degree of violation of the Lam-Tung relation, $1-\lambda-2 v$, for the E615 experiment as well as predictions for the COMPASS experiment. Many salient features of transverse momentum and rapidity dependence could be qualitatively understood by a geometric approach.

XXVII International Workshop on Deep-Inelastic Scattering and Related Subjects - DIS2019 8-12 April, 2019

Torino, Italy

\footnotetext{
* Speaker.
} 


\section{Introduction}

The Drell-Yan (D-Y) process together with deep inelastic scattering are the main tools for extracting the parton distributions in hadrons [W]. The polar and azimuthal angular distributions of leptons produced in unpolarized D-Y process are sensitive to the underlying reaction mechanisms as well as novel parton distributions. For example, Boer-Mulders functions [ [ $]$ ] have been suggested to account for a violation of the Lam-Tung (L-T) relation [B] observed in the fixed-target experiments with pion beams, e.g. E615 [四].

In this proceedings, we compare the data of dilepton angular parameters $\lambda, \mu, v$ and the L-T violation quantity $1-\lambda-2 v$ measured by E615 [䧃] with the fixed-order pQCD calculations [ []]. Furthermore we present the NLO pQCD predictions for the ongoing COMPASS [目] experiments on the dimuon mass $Q$ and Feynman- $x\left(x_{F}\right)$ dependence of these angular parameters. In addition,

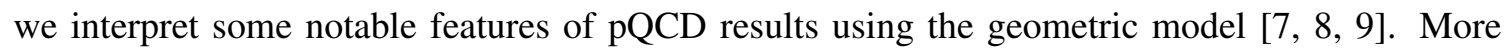
results and greater details can be found in Ref. [ए]].

\section{Calculations of dilepton angular parameters in DYNNLO}

We utilize the DYNNLO (version 1.5) package for the calculations. Via the LHAPDF6 framework, the parton distribution functions (PDFs) used for the protons and neutrons are "CT14nlo" and "CT14nnlo" in the NLO and NNLO calculations, respectively, and "GRVPI1" for the pion PDFs in both NLO and NNLO calculations.

In the rest frame of the virtual photon in the D-Y process, a commonly used expression for the lepton angular distributions is given as

$$
\frac{d \sigma}{d \Omega} \propto 1+\lambda \cos ^{2} \theta+\mu \sin 2 \theta \cos \phi+\frac{v}{2} \sin ^{2} \theta \cos 2 \phi,
$$

where $\theta$ and $\phi$ refer to the polar and azimuthal angles of $l^{-}\left(e^{-}\right.$or $\left.\mu^{-}\right)$. To obtain the $\lambda, \mu$, and $v$ parameters, we first calculate the $A_{i}$ parameters in an alternative expression of the lepton angular distributions of the $\mathrm{D}-\mathrm{Y}$ process as follows:

$$
\frac{d \sigma}{d \Omega} \propto\left(1+\cos ^{2} \theta\right)+\frac{A_{0}}{2}\left(1-3 \cos ^{2} \theta\right)+A_{1} \sin 2 \theta \cos \phi+\frac{A_{2}}{2} \sin ^{2} \theta \cos 2 \phi .
$$

The angular coefficients $A_{i}$ could be evaluated by the moments of spherical harmonic polynomial expressed as

$$
A_{0}=4-10\left\langle\cos ^{2} \theta\right\rangle ; \quad A_{1}=5\langle\sin 2 \theta \cos \phi\rangle ; \quad A_{2}=10\left\langle\sin ^{2} \theta \cos 2 \phi\right\rangle,
$$

where $\langle f(\theta, \phi)\rangle$ denotes the moment of $f(\theta, \phi)$, i.e. the weighted average of $f(\theta, \phi)$ by the cross sections in Eq. (2.2). It is straightforward to show that $\lambda, \mu, v$ in Eq. (2.]) are related to $A_{0}, A_{1}, A_{2}$ via

$$
\lambda=\frac{2-3 A_{0}}{2+A_{0}} ; \quad \mu=\frac{2 A_{1}}{2+A_{0}} ; \quad v=\frac{2 A_{2}}{2+A_{0}} .
$$

Equation (2.4) shows that the L-T relation, $1-\lambda-2 v=0$, is equivalent to $A_{0}=A_{2}$. 
In Fig. 田, we compare the results of $\lambda, \mu, v$, and the L-T violation, $1-\lambda-2 v$, from the fixedorder pQCD calculations with $252-\mathrm{GeV} \pi^{-}+W$ data from E615 experiment [四]. The angular parameters are evaluated as a function of the dimuon's transverse momentum $\left(q_{T}\right)$ in the CollinsSoper frame. Overall, the calculated $\lambda, \mu$ and $v$ exhibit distinct $q_{T}$ dependencies. At $q_{T} \rightarrow 0, \lambda, \mu$ and $v$ approach the values predicted by the collinear parton model: $\lambda=1$ and $\mu=v=0$. As $q_{T}$ increases, Fig. $\square$ shows that $\lambda$ decreases toward its large- $q_{T}$ limit of $-1 / 3$ while $v$ increases toward $2 / 3$, for both $q \bar{q}$ and $q G$ processes [ [W, [2]]. The $q_{T}$ dependence of $\mu$ is relatively mild compared to $\lambda$ and $v$. This is understood as a result of some cancellation effect, to be discussed in Sec. [].

Comparing the results of the NLO with the NNLO calculations, $\lambda$ (NNLO) is smaller than $\lambda$ (NLO) while $\mu$ and $v$ are very similar for NLO and NNLO. The amount of L-T violation, $1-$ $\lambda-2 v$, is zero in the NLO calculation, and nonzero and positive in the NNLO calculation. As seen in Fig. I, the pQCD predicts a sizable magnitude for $v$, comparable to the data. Therefore, the pQCD effect should be included in the extraction of nonperturbative Boer-Mulders effect from the data of $v$.

$\mathrm{E} 615 \pi^{-}+\mathrm{W}$ at $252 \mathrm{GeV}$
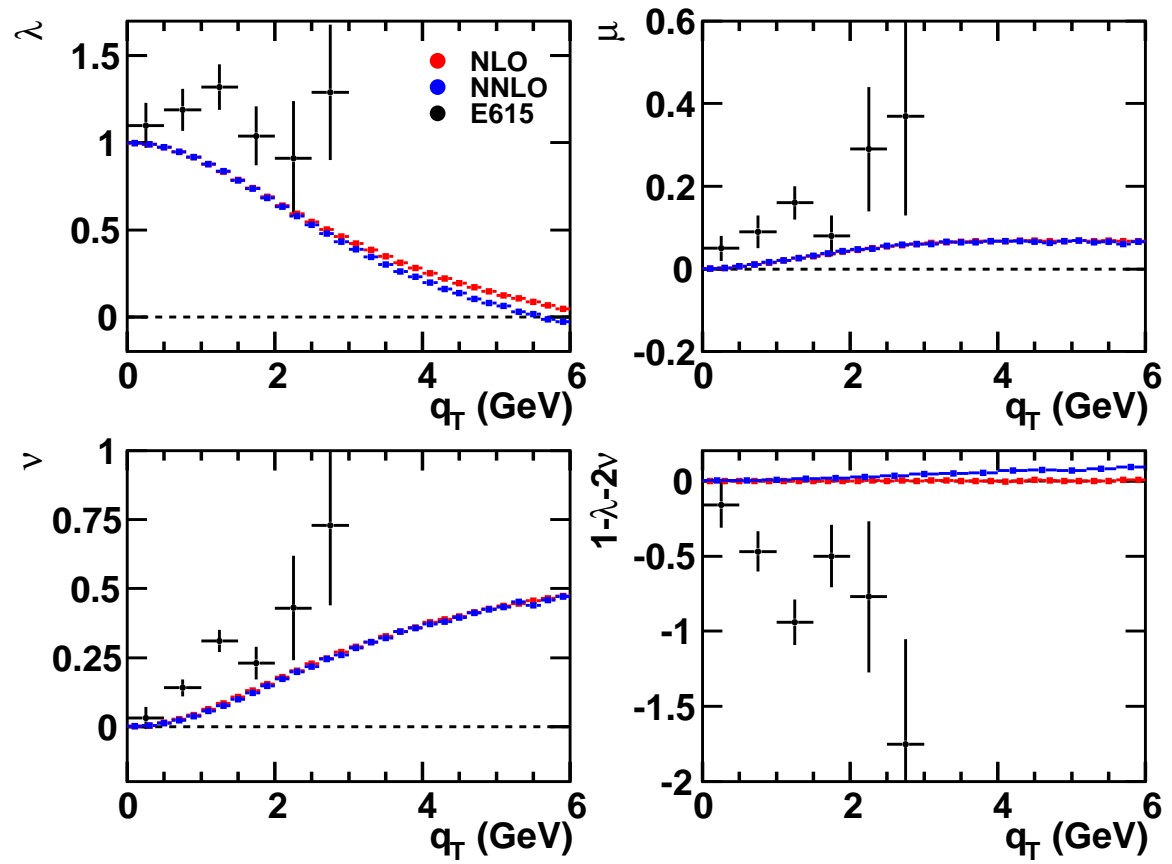

Figure 1: Comparison of NLO (red points) and NNLO (blue points) fixed-order pQCD calculations with the $\mathrm{E} 615 \pi^{-}+W \mathrm{D}-\mathrm{Y}$ data at $252 \mathrm{GeV}$ [䧃] (black points) for $\lambda, \mu, v$ and $1-\lambda-2 v$.

Next we present the results of the angular coefficients $\lambda, \mu$ and $v$ as a function of $q_{T}$ in various bins of $Q$ and $x_{F}$ for the ongoing fixed-target COMPASS experiment at CERN [G]. This experiment runs with $190-\mathrm{GeV} \pi^{-}$beam and transversely-polarized $\mathrm{NH}_{3}$ target and unpolarized aluminum $(\mathrm{Al})$ and tungsten $(W)$ nuclear targets. There are three bins for $Q$ in the range of 4.0-7.0 GeV, as well as three bins for $x_{F}$ in the range of $0-0.6$. Our results could be convoluted by the COMPASS spectrometer acceptances later for a direct comparison with experimental data in the near future. Since there are no significant difference between the NLO and NNLO results, we present only the 
results from the NLO calculation to illustrate the major features.

Figures $\square(a)$ and (b) show $\lambda, \mu$ and $v$ as a function of $q_{T}$ for various bins of the dimuon mass, $Q$, and Feynman- $x, x_{F}$, respectively. The $q_{T}$ distributions of $\lambda$ and $v$ parameters depend sensitively on $Q$, but only weakly on $x_{F}$. As for $\mu$, its $q_{T}$ distribution has strong dependencies on $x_{F}$ and on $Q$. In particular, the magnitude of $\mu$ is small when $x_{F}$ is close to 0 and its sign could even turn negative at some $q_{T}$ region. As $x_{F}$ increases, the magnitude of $\mu$ increases pronouncedly.

\section{Geometric model}

The E615 D-Y data of lepton angular distributions can be reasonably well described by the NLO and NNLO pQCD calculations. Various salient features of $Q$ and $x_{F}$ dependencies are observed in the predicted results of $\lambda, \mu$ and $v$ parameters for COMPASS experiment based on NLO pQCD. It is of interest to check if these features of pQCD calculations could be understood using the geometric approach developed in Refs. [ [, , 8].

In Refs. [ $[\mathbb{Z}, \mathbb{8}]$, the hadron plane, the quark plane, and the lepton plane of collision geometry are defined in the Collins-Soper $\gamma^{*}$ rest frame. A pair of collinear $q$ and $\bar{q}$ with equal momenta annihilate into a $\gamma^{*}$. The momentum unit vector of $q$ is defined as $\hat{z}^{\prime}$, and the quark plane is formed by the $\hat{z}^{\prime}$ and the $\hat{z}$ axes of Collins-Soper frame. The angular coefficients $A_{i}$ in Eq. (L2.2) can be expressed in term of $\theta_{1}$ and $\phi_{1}$ as follows:

$$
A_{0}=\left\langle\sin ^{2} \theta_{1}\right\rangle, \quad A_{1}=\frac{1}{2}\left\langle\sin 2 \theta_{1} \cos \phi_{1}\right\rangle, \quad A_{2}=\left\langle\sin ^{2} \theta_{1} \cos 2 \phi_{1}\right\rangle,
$$

where the $\theta_{1}$ and $\phi_{1}$ are the polar and azimuthal angles of the natural quark axis $\hat{z}^{\prime}$ of the quark plane in the Collins-Soper frame. The $\langle\cdots\rangle$ in Eq. (B.]) denotes that the measured values of $A_{i}$ at a given kinematic bin are averaged over events having particular values of $\theta_{1}$ and $\phi_{1}$.

Up to NLO $\left(\mathscr{O}\left(\alpha_{S}\right)\right)$ in pQCD, the quark plane coincides with the hadron plane and $\phi_{1}=$ 0 . Therefore $A_{0}=A_{2}$ or $1-\lambda-2 v=0$, i.e., the L-T relation is satisfied. Higher order pQCD processes allow the quark plane to deviate from the hadron plane, i.e., $\phi_{1} \neq 0$. This acoplanarity effect leads to the violation of the L-T relation. For a nonzero $\phi_{1}$, Eq. (B.D) shows that $A_{2}<$ $A_{0}$. Therefore, when the L-T relation is violated, $A_{0}$ must be greater than $A_{2}$ or, equivalently, $1-\lambda-2 v>0$. This expectation of $1-\lambda-2 v>0$ in the geometric approach agrees with the results of NNLO pQCD calculations shown in Fig. $\mathbb{\text { ll }}$. The geometric approach offers a simple interpretation for this result.

Furthermore the sign of $\mu$ could be either positive or negative, depending on which parton and hadron the gluon is emitted from [ [8, [0]]. Hence, one expects some cancellation effects for $\mu$ among contributions from various processes. Each process is weighted by the corresponding density distributions for the interacting partons. At $x_{F} \sim 0$, the momentum fraction carried by the beam parton $\left(x_{B}\right)$ is comparable to that of the target parton $\left(x_{T}\right)$. Therefore, the weighting factors for various processes are of similar magnitude and the cancellation effect could be very significant, resulting in a small value of $\mu$. As $x_{F}$ increases toward $1, x_{B}$ becomes much larger than $x_{T}$. In this case the weighting factors are now dominated by fewer processes, resulting in less cancellation and a larger value of $\mu$. This explains why the $\mu$ parameter exhibits a strong $x_{F}$ dependence in Figs. D(b). 


\section{COMPASS $\pi^{-}+W$ at $190 \mathrm{GeV}$}
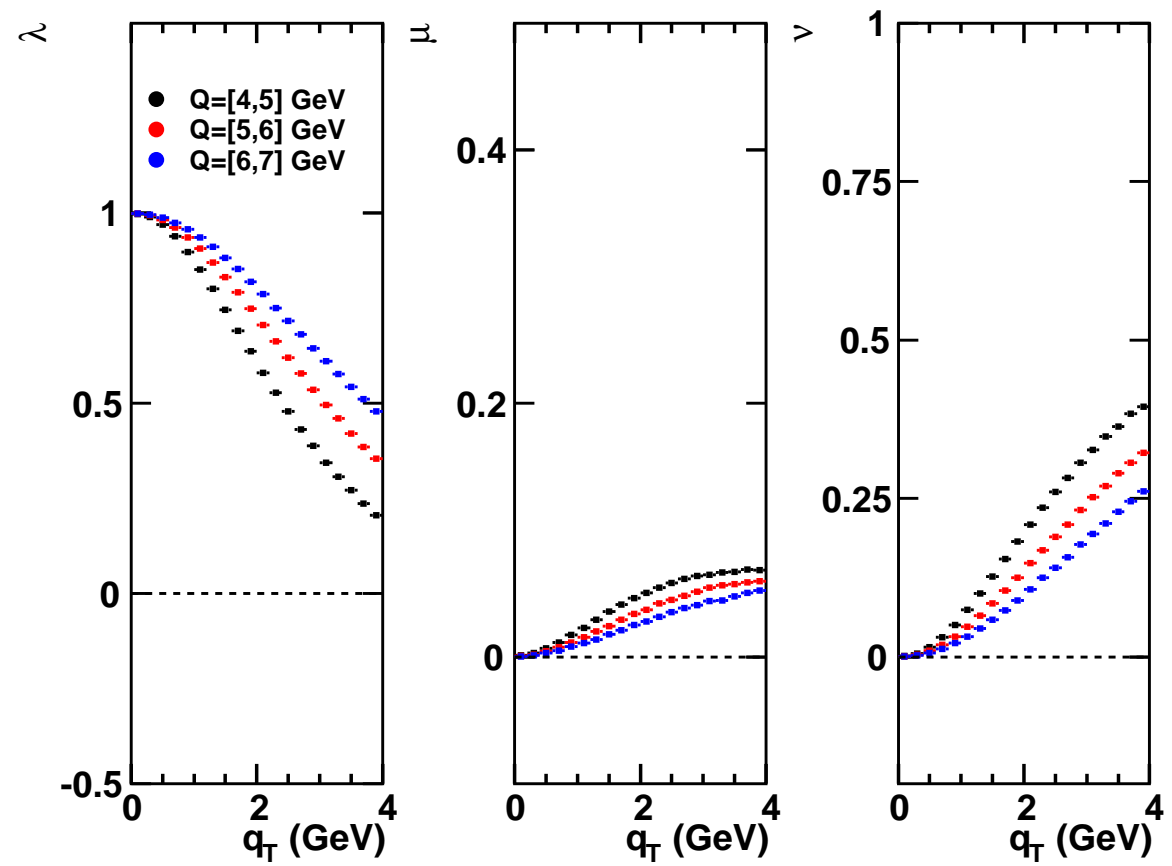

(a)

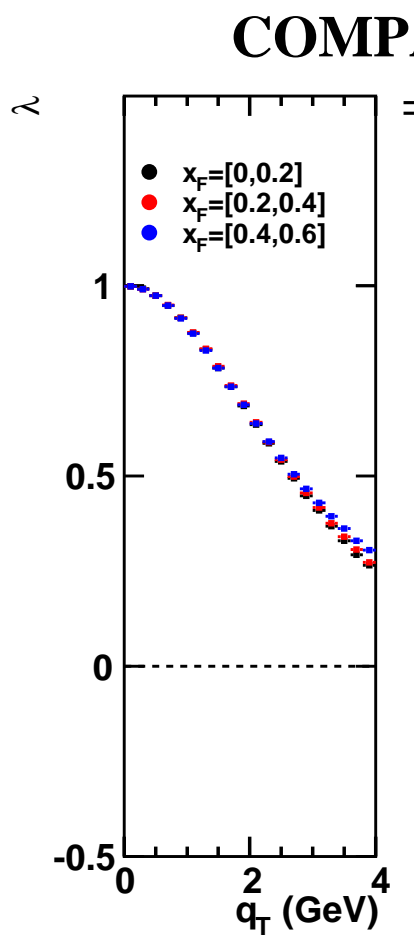

ASS $\pi^{-}+W$ at $190 \mathrm{GeV}$
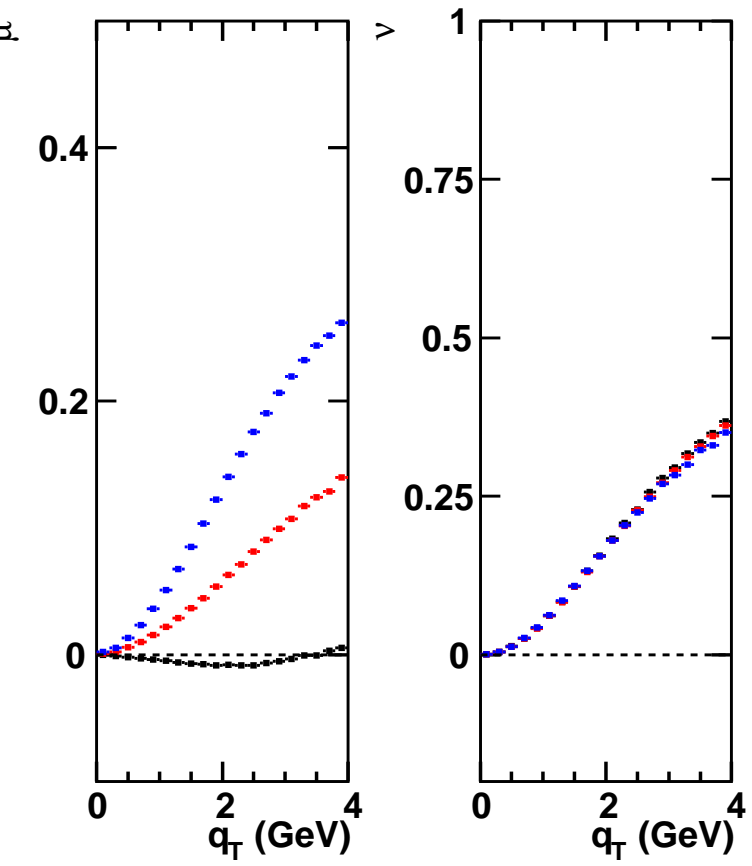

(b)

Figure 2: (a) NLO pQCD results of $\lambda, \mu$, and $v$ as a function of $q_{T}$ at several dimuon mass $Q$ bins and $x_{F}>0$ for D-Y production off the tungsten target with $190-\mathrm{GeV} \pi^{-}$beam in the COMPASS experiment. (b) Same as (a) but at several Feynman- $x x_{F}$ bins and $4<Q<9 \mathrm{GeV}$. 


\section{Summary}

We have presented a comparison of the measurements of the angular parameters $\lambda, \mu, v$ and $1-\lambda-2 v$ of the D-Y process from the fixed-target E615 experiment with the corresponding results from the NLO and NNLO pQCD calculations. Qualitatively the transverse momentum $\left(q_{T}\right)$ dependence of $\lambda, \mu$ and $v$ in the data could be described by pQCD. The difference between NLO and NNLO results becomes visible at large $q_{T}$. The L-T violation part $1-\lambda-2 v$ remains zero in the NLO pQCD calculation and turns positive in NNLO pQCD.

The $x_{F}$ dependence of the angular parameters is well described by the geometric picture. In particular, the weak rapidity dependencies of the $\lambda$ and $v$, and the pronounced rapidity dependency for $\mu$ can be explained by the absence or presence of rapidity-dependent cancellation effects. The occurrence of acoplanarity between the quark plane and the hadron plane $\left(\phi_{1} \neq 0\right)$, for the pQCD processes beyond NLO leads to a violation of the L-T relation. The predicted positive value of $1-\lambda-2 v$, or $A_{0}>A_{2}$ when $\phi_{1}$ is nonzero, is consistent with the NNLO pQCD results.

\section{References}

[1] J. C. Peng and J. W. Qiu, Prog. Part. Nucl. Phys. 76, 43 (2014).

[2] D. Boer, Phys. Rev. D 60, 014012 (1999).

[3] C. S. Lam and W. K. Tung, Phys. Rev. D 21, 2712 (1980).

[4] E615 Collaboration, J. S. Conway et al., Phys. Rev. D 39, 92 (1989); J. G. Heinrich et al., Phys. Rev. D 44, 1909 (1991).

[5] M. Lambertsen and W. Vogelsang, Phys. Rev. D 93, 114013 (2016).

[6] COMPASS-II Proposal, Report No. CERN-SPSC-2010; M. Aghasyan et al. (COMPASS Collaboration), Phys. Rev. Lett. 119, 112002 (2017).

[7] J. C. Peng, W. C. Chang, R. E. McClellan, and O. Teryaev, Phys. Lett. B 758, 384 (2016).

[8] W. C. Chang, R. E. McClellan, J. C. Peng and O. Teryaev, Phys. Rev. D 96, 054020 (2017).

[9] J. C. Peng, D. Boer, W. C. Chang, R. E. McClellan and O. Teryaev, Phys. Lett. B 789, 356 (2019).

[10] W. C. Chang, R. E. McClellan, J. C. Peng and O. Teryaev, Phys. Rev. D 99, no. 1, 014032 (2019).

[11] R. L. Thews, Phys. Rev. Lett. 43, 987 (1979).

[12] J. Lindfors, Phys. Scr. 20, 19 (1979). 